\title{
Disruption of Saccadic Adaptation with Repetitive Transcranial Magnetic Stimulation of the Posterior Cerebellum in Humans
}

\author{
Ned Jenkinson $\cdot$ R. Chris Miall
}

Published online: 28 July 2010

(C) The Author(s) 2010. This article is published with open access at Springerlink.com

\begin{abstract}
Saccadic eye movements are driven by motor commands that are continuously modified so that errors created by eye muscle fatigue, injury, or - in humans-wearing spectacles can be corrected. It is possible to rapidly adapt saccades in the laboratory by introducing a discrepancy between the intended and actual saccadic target. Neurophysiological and lesion studies in the non-human primate as well as neuroimaging and patient studies in humans have demonstrated that the oculomotor vermis (lobules VI and VII of the posterior cerebellum) is critical for saccadic adaptation. We studied the effect of transiently disrupting the function of posterior cerebellum with repetitive transcranial magnetic stimulation (rTMS) on the ability of healthy human subjects to adapt saccadic eye movements. rTMS significantly impaired the adaptation of the amplitude of saccades, without modulating saccadic amplitude or variability in baseline conditions. Moreover, increasing the intensity of rTMS produced a larger impairment in the ability to adapt saccadic size. These results provide direct evidence for the role of the posterior cerebellum in man and further evidence that TMS can modulate cerebellar function.
\end{abstract}

N. Jenkinson

Nuffield Department of Surgery, University of Oxford,

Oxford, UK

R. C. Miall

School of Psychology, University of Birmingham,

Birmingham, UK

\section{N. Jenkinson $(\square)$}

West Wing, John Radcliffe Hospital,

Oxford Functional Neurosurgery, Level 6 Headley Way,

OX3 9DU Oxford, UK

e-mail: ned.jenkinson@dpag.ox.ac.uk
Keywords Cerebellum · Saccades · Adaptation · Physiological $\cdot$ Eye movements/physiology Transcranial magnetic stimulation $\cdot$ Repetitive

\section{Introduction}

Several areas of cerebral cortex contribute to the production of motor commands for saccadic eye movements in humans; these include the frontal and supplementary eye fields and the dorsolateral prefrontal cortex. Although these areas can be responsible for the generation of saccades, they do not produce the movements directly. Cortical areas send efferent connections, to a large extent, via the superior colliculus to the premotor burst neurones (PBNs) and omnipause neurones that directly influence eye movements. The other major influence on saccadic generation is the cerebellum. Stimulation of several parts of the cerebellum can evoke saccades [1]. Noda later defined a saccadic area within cerebellar lobules VI and VII now known as the oculomotor vermis (OMV) [2, 3]. Purkinje cells of the OMV inhibit neurones in the caudal fastigial nuclei (CFN), which in turn projects to the PBNs. Timing of activity of CFN neurones has been demonstrated to vary with saccadic size and is thought to control the size of saccades via its influence on the PBNs $[4,5]$. If the CFN is destroyed with [6,7] or without [8] the overlying cerebellar cortex, saccades become permanently hypermetric, and the ability to adapt saccadic amplitude is lost. Similar results are found if the $\mathrm{CFN}$ is inactivated with muscimol, though latent adaptation is revealed once the inactivation effects wear off, suggesting that the adaptation is taking place at a locus upstream of the CFN [9]. A likely candidate is the OMV, and it has been shown that lesions of the OMV that spare the deep nuclei permanently abolish the ability to rapidly adapt the amplitude of saccades $[10,11]$. 
The cerebellum has long been implicated in motor learning in the vestibulo-ocular [12, 13] and blink reflexes [14, 15] as well as in the visuomotor system [16, 17]. Marr [18] and Albus [19] famously suggested a model of cerebellar motor learning based on Purkinje cell output modulated by simple and complex spike inputs from mossy and climbing fibre systems, respectively. The exact role of the climbing fibre input to the cerebellum is not clear with some studies, suggesting that its role is to provide a corrective error signal [20], and others proposing that the signal drives the stabilisation of newly learnt motor behaviours [21]. Regardless of the precise role of the climbing fibre input to the OMV, it is generally agreed that this region of the cerebellum plays a crucial role in saccadic plasticity and that changes in the timing of the firing of climbing fibres in the OMV is associated with saccadic adaptation. Furthermore, it is thought that, of the two broad categories of saccades (reactive saccades and voluntary saccades), the cerebellum is likely to be the site of saccadic adaptation of reactive pro-saccades, whereas other more voluntary saccades are likely to have a site of adaption upstream in the cortex (see [22]).

For these reasons, we used 1-Hz rTMS to interfere with the posterior cerebellum including the OMV during a reactive pro-saccadic adaptation task to demonstrate that this area of the cerebellum is critical to adaptation of this saccade type.

\section{Materials and Methods}

\section{Subjects}

Nineteen healthy volunteers (four females, 15 males; mean age, 32.3 years; SEM, 2.2) participated in this study after giving informed written consent. They were randomly assigned to one of three subject groups. The experiment was performed in compliance with relevant institutional guidelines and approved by the local ethics committee. One subject was omitted from the final analysis due to poorquality erratic saccadic behaviour; a further two did not finish the behavioural testing because TMS over the back of the head produced too much head movement. The data from these three subjects were not included in any of the analysis in this study; accordingly, the group sizes do not include these subjects.

\section{Eye Movement Recordings}

Subjects were placed on a chin rest and held in position with the TMS double-cone coil on a mechanical arm at the centre an arc of 40 red light-emitting diodes (LEDs), at a radius of $57 \mathrm{~cm}$ in a dark room. Horizontal eye position of the right eye was monitored using an infrared light eye tracker (Skalar medical, Delft, Netherlands) at a spatial resolution of $0.5^{\circ}$. An eye patch was worn over the left eye. The data were sampled at a frequency of $250 \mathrm{~Hz}$ and stored on a computer for off-line analysis. Calibrations were performed by asking the subjects to fixate on five targets at $-12^{\circ},-6^{\circ}, 0^{\circ}, 6^{\circ}$ and $12^{\circ}$ from the midline. Calibrations were taken before the start and end of all eye-tracking sessions; an additional calibration was performed at the midpoint of the 100 trial adaptation sessions.

\section{Pre-adaptation Task}

Each trial in the pre-adaptation task started with the illumination of an initial fixation LED. To avoid subjects making saccades to remembered targets, the initial fixation LEDs' position varied randomly across $-10^{\circ},-8^{\circ}$ and $-6^{\circ}$ from the midline. The start position was offset so that the eye movements took place within the calibration targets. All target positions were relative to the initial fixation position. The initial fixation target was accompanied by a tone to indicate the beginning of a trial. After fixation on the start LED, subjects were encouraged to blink if necessary, before self-initiating the trial by pressing a computer key. They were required to maintain fixation until the initial fixation LED was extinguished 500 to $1,500 \mathrm{~ms}$ later. At this point, a new target LED $16^{\circ}$ to the left of the fixation point was illuminated. The LED remained illuminated until the subject's eye position was fixated stably within $2^{\circ}$ of the new target for a period of $200 \mathrm{~ms}$. The target LED was then extinguished, and the next central fixation target illuminated once the eye position returned to within $5^{\circ}$ of the starting position, accompanied by a tone to indicate the beginning of the next trial. All subjects completed at least 10 trials in these conditions to calculate the pre-adaptation primary saccade amplitude, before receiving rTMS.

\section{Induction of Saccadic Adaptation}

Saccadic eye movements can be rapidly adapted using a simple experimental technique that feeds errors into the saccadic system, thereby forcing inaccurate saccades [23]. In the adaptation task, the conditions were identical to the pre-adaptation task until the first data sample when the eye position had left the zone approximately $5^{\circ}$ around the extinguished initial fixation point, at which point the target LED at $16^{\circ}$ was extinguished and a second target LED $10^{\circ}$ to the left of the fixation position was illuminated. This second LED remained illuminated until the eye position was stable within $2^{\circ}$ for a period of $200 \mathrm{~ms}$. The final position target LED was then extinguished, and the next start fixation target illuminated once the eye position returned to within $5^{\circ}$ of the previous start position, accompanied by a tone to indicate the start of the next trial. The room was in darkness for $2 \mathrm{~min}$ prior to the start of the adaptation session and 
throughout the 100 saccadic trials (approximately 8-min duration). The subjects were encouraged to make rapid accurate eye movements to the new target as soon as it was illuminated. No subject reported perceiving any displacement of the initial target during the saccade.

\section{Repetitive Transcranial Magnetic Stimulation}

Low-frequency $1-\mathrm{Hz}$ rTMS temporarily disrupts neural activity by delivering repetitive focal magnetic pulses to specific regions of the brain [24]. Because there is no direct way to determine the stimulus level needed to affect the cerebellum, three treatment groups were used, one receiving no TMS $(n=7)$ and the other two receiving either $45 \%$ $(n=6)$ or $55 \%(n=6)$ of machine output. TMS was delivered using a Magstim Rapid stimulator (Magstim Company Ltd., Whitland, Wales, UK) through a doublecone coil placed firmly centred over the inion and directed at the posterior cerebellum. We chose this location after examining several magnetic resonance imagings (MRIs) of randomly chosen heads that indicated that stimulation at this point would target the posterior cerebellum (see Fig. 1). Current direction in the coil was downward which produces an upward current in the target region that is thought to be best suited to stimulating the cerebellum [25]. All subjects were given 120 TMS pulses at a frequency of $1 \mathrm{~Hz}$ following the pre-adaptation task and before the onset of the saccadic adaptation paradigm. This number of $1-\mathrm{Hz}$ pulses has been shown to produce a significant reduction of excitability in the neural tissue at which it is directed [26]. Repetitive TMS continued throughout the adaptation paradigm, at a base rate of $1 \mathrm{~Hz}$. However, to avoid possible direct TMS-induced perturbation of the ongoing saccades, the computer monitoring the eye movements was programmed so that a TMS pulse due to be delivered during the reaction time interval between the presentation of the first or second target LEDs (i.e. in the reaction and saccadic movement time) was delayed until the end of the eye movement. This resulted in a delay of approximately $200-300 \mathrm{~ms}$ on a low proportion of trials; the subsequent TMS interval was maintained at $1,000 \mathrm{~ms}$. Estimated frequency of stimulation across the whole stimulation period was $0.97-1.0 \mathrm{~Hz}$.

\section{Data Analysis}

We calculated the amount of adaptation taking place in an individual in the 100 adaptation trials as the difference between the mean of the final 10 saccades in the pre-adaptation trials and the mean of the final 10 initial saccades of the adaptation
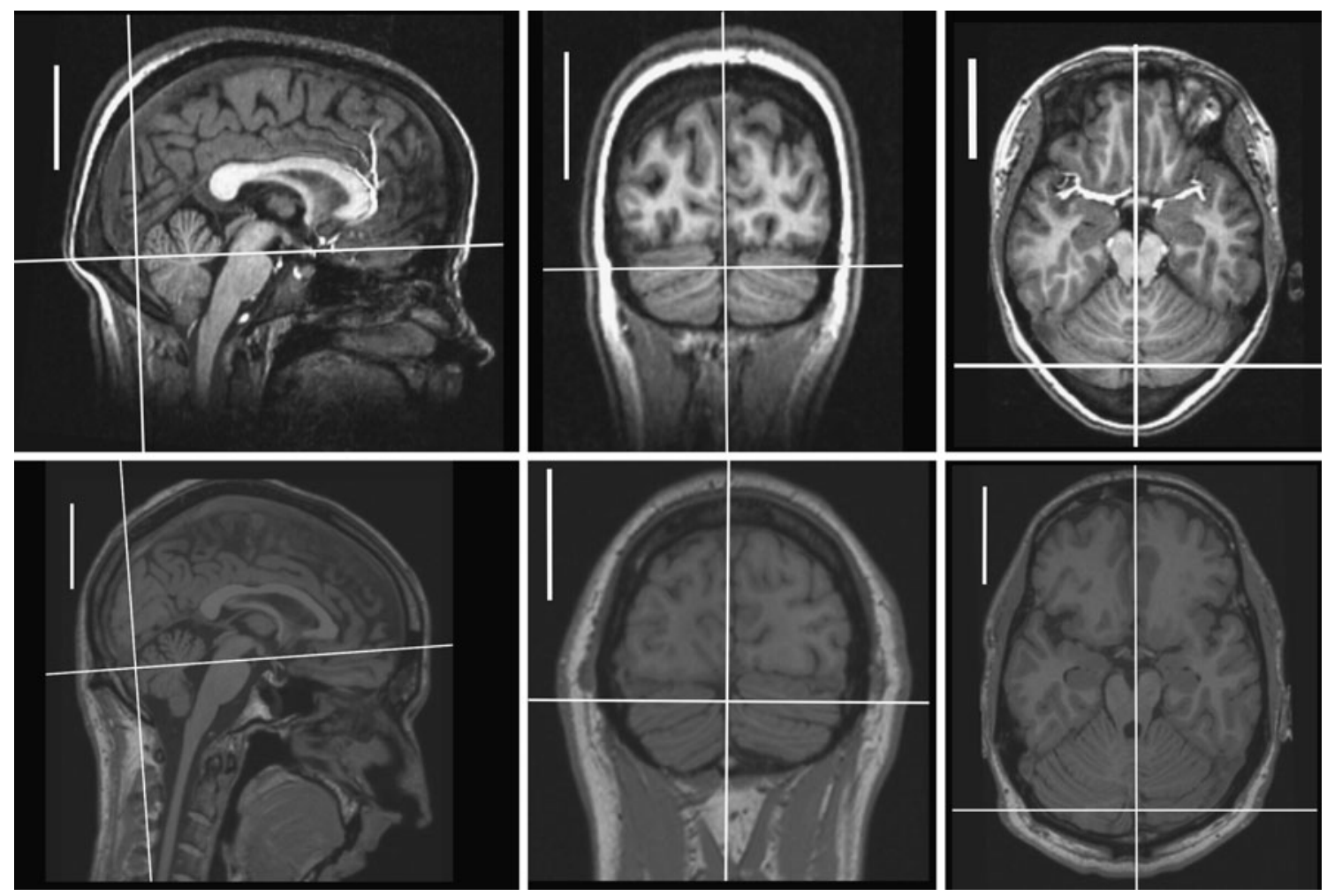

Fig. 1. Site of rTMS stimulation. MRIs of two of the subjects in the study showing the site stimulated; the white crosshairs represent the intended target of stimulation. Note the position of the visual cortex in relation to the inion. There is a 5-cm white scale bar to the left side of each image 
trial. Initial saccades were taken as the first large saccade toward the first target. Secondary saccades toward the target due to dysmetria of the initial saccade were ignored. To remove any outliers, we iteratively fitted an exponential curve to the adaptation data within each session and removed any saccades with amplitude of $2 \mathrm{SD}$ away from the curve.

\section{Results}

\section{Effect of rTMS on Saccadic Adaptation}

Low-frequency rTMS over the OMV of the cerebellum reduces the magnitude of adaptation of saccadic eye movements in the normal healthy human subject. Preadaption accuracy was not significantly different across groups (Fig. $2 ; F_{2}, 16=0.47, p=0.63$ ). For each individual, the amount of adaptation was calculated as the difference between the mean pre-adaptation saccadic amplitude and the mean amplitude of the last 10 saccades. A one-way analysis of variance (ANOVA) revealed that the amount of adaptation was significantly different across the three groups $\left(F_{2,16}=12.09, p<0.01\right)$. Tukey post hoc comparisons found that the $55 \%$ group showed significantly less adaptation than the other two groups $(55 \%$ vs. $0 \%, p<$ $0.001 ; 55 \%$ vs. $45 \% p<0.05)$ who were not significantly different $(45 \%$ vs. $0 \% p=0.12)$. The group that produced the most adaptation was that which received no rTMS $(0 \%)$; this group reduced their mean saccadic amplitude from $15.36^{\circ}$ to $10.52^{\circ}$ (Fig. 2; top panel, right-hand side), which represents $84 \%$ of the ideal gain decrease of 0.375 . For the $0 \%$ group, the mean difference between the preadaptation and final saccadic size is $4.84^{\circ}\left(\mathrm{SEM}, 0.36^{\circ}\right)$. The groups that demonstrated the least adaptation were those that had the highest level of TMS. The 55\% rTMS group reduced their mean initial saccade size from $15.61^{\circ}$ to $13.17^{\circ}$; only $41 \%$ of the ideal gain reduction (Fig. 2; bottom panel, right-hand side). For this group, the mean difference between pre-adaptation and final saccadic size is $2.44^{\circ}$ (SEM, $0.43^{\circ}$ ). For the $45 \%$ TMS group, their initial amplitude was $15.25^{\circ}$, with a mean final saccadic amplitude of $11.45^{\circ}$ or $66 \%$ of the ideal gain reduction (Fig. 2; middle panel, right-hand side). The mean difference for this group between the pre-adaptation and the final amplitude is $3.80^{\circ}$ (SEM, $0.20^{\circ}$ ). The left side of Fig. 2 shows the baseline saccades and the different time courses of saccadic adaptation for all individuals in each group: the no rTMS (top), 45\% rTMS (middle) and 55\% rTMS (bottom). To test whether rTMS caused a change in the variability of the initial saccade, we calculated the standard error around the mean of the last 10 saccades for each individual in each group. There was no difference in the individual measures of variability across the three groups $\left(F_{2,16}=0.78, p=0.93\right)$.

\section{Effect of rTMS on Saccade Peak Velocity}

We calculated the mean peak velocity of the first and last 10 saccades in each adaptation session for each individual in each group. A one-way ANOVA revealed that there was no significant difference between the initial peak velocities between the groups $\left(F_{2}, 16=2.09, p=0.16\right)$. However, the groups' final peak velocities were just significantly different $\left(F_{2}, 16=3.67, p=0.049\right)$, with post hoc Tukey tests revealing a difference only between the $0 \%$ and $55 \%$ group, a decrease in peak velocity of $-23 \%$ and $-11 \%$, respectively, thus replicating the pattern of changes seen in amplitude.

\section{Effect of rTMS on Normal Saccades}

To ensure that the rTMS in itself did not affect the ability of an individual to perform saccades, we had two subjects make repeated eye movements to a fixed $16^{\circ}$ target (see preadaptation task) for 100 trials with $45 \%$ rTMS directed at the posterior cerebellum before the start and throughout the trials (see "Methods: TMS adaptation trials"). The mean saccadic amplitudes for the first and last 10 saccades of all 100 trials were $15.63^{\circ}$ and $15.70^{\circ}$, respectively. There was also no effect of TMS on the number of corrective saccades, with one subject making 51 corrective saccades (out of 100 saccades) without TMS and 53 with TMS and the other subject making 48 and 50, respectively. The accuracy of the initial saccade during both the TMS and non-TMS sessions suggests that the target is accurately localised, with no hypometria of saccades following TMS, unlike that seen after physical ablation of the OMV $[10,27]$. There was however a small $(8 \%)$ reduction in mean peak velocity.

\section{Discussion}

We investigated whether rTMS of the oculomotor cerebellum would interfere with the ability of healthy human subjects to adapt the size of their saccades. We used a standard inward saccadic adaptation task and applied two intensities of rTMS to the posterior cerebellum in comparison to subjects performing the same task without rTMS. rTMS over the posterior cerebellum reduced the ability to alter the size of saccades. The amount of adaptation decreased with an increase in the intensity of rTMS. Thus, the effect of rTMS over the cerebellum is to temporarily reduce the plasticity of the oculomotor system, and we suggest that this is due to disruption of the normal function of the OMV. Our finding is consistent with the existing literature that show that surgical lesions in non-human primates and pathological lesions in humans produce a similar deficit in saccadic adaptation $[10$, 27-29], and single-cell studies that show that Purkinje cells 
No TMS
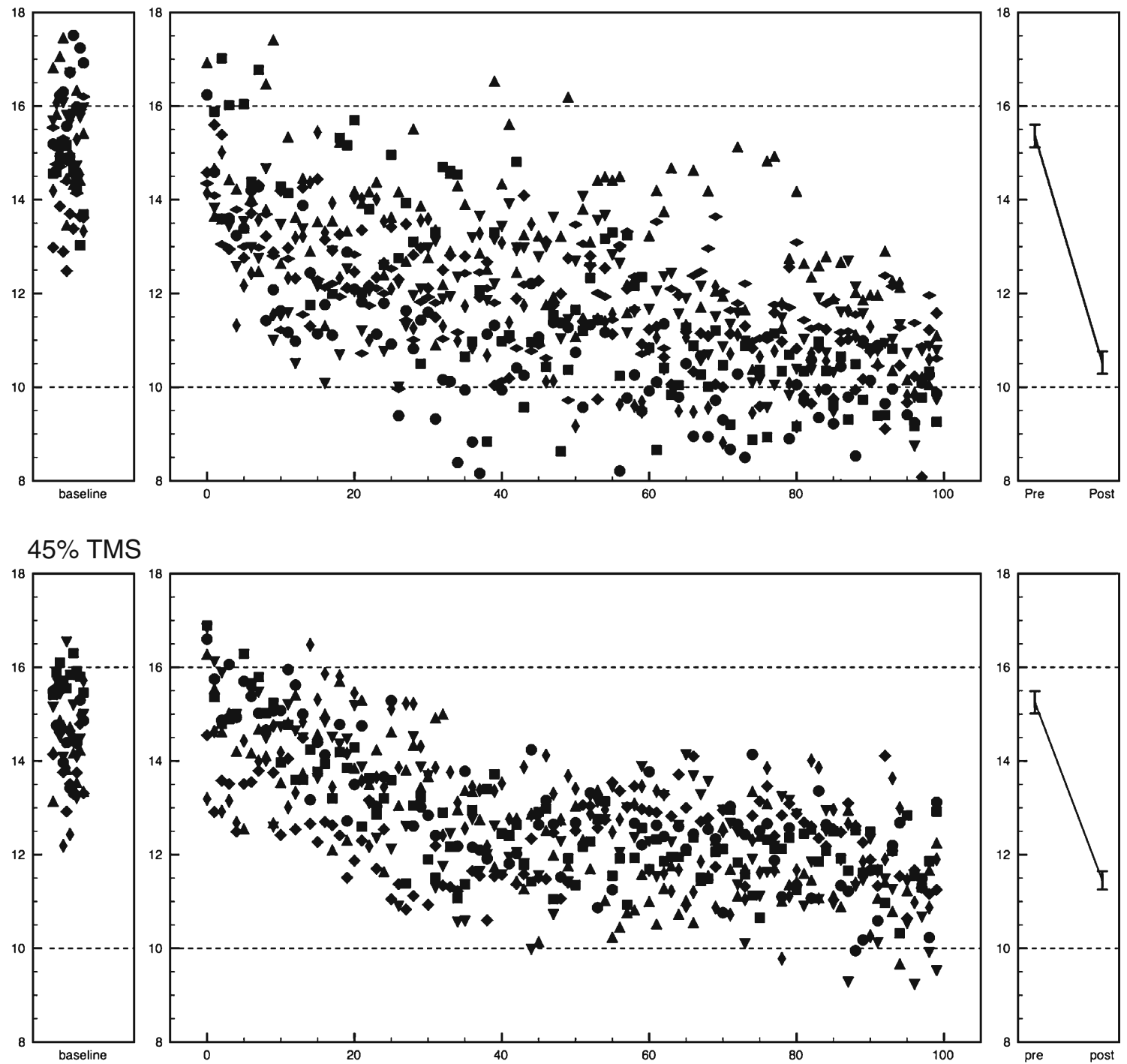

$55 \%$ TMS
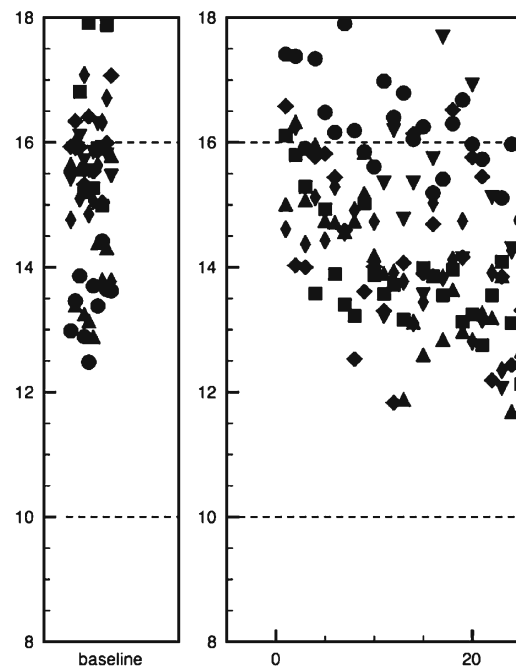
4Fig. 2. Individual and mean results of the three groups performing the saccadic adaptation task. The line plots on the right-hand side of each panel show the mean saccadic amplitude of the 10 pre-adaptation baseline saccades made to a $16^{\circ}$ target and the mean saccadic amplitude of the last 10 saccades of the adaptation experiment; error bars represent the standard error of the means. The scatter plots on the left of each panel represent the trial-by-trial amplitude of the primary reactive saccade for each individual in the group, after exclusion of outliers. Different symbols are used for each participant in each group. The left-most data are for baseline trials in which participants moved towards static targets; the central data points are the primary saccades made by each individual during the time course of 100 adaptive trials. Horizontal dotted lines indicate the amplitude of the ideal initial and final saccades

in the OMV alter their firing rates in a way that could alter saccadic amplitude [20,30, 31].

What evidence is there that TMS can modulate the function of the OMV of the cerebellum? Single pulses of TMS over the cerebellum have also been demonstrated to disrupt visually guided saccades [32] and smooth pursuit eye movements [33], as well as coordinated eye and head movements [34], and more complex cerebellar functions such as state estimation [35]. Consistent with cerebellothalamo-cortical connections, several studies to date have demonstrated that a single pulse of TMS over the lateral cerebellum can be used to condition motor cortical excitability, as measured by the response elicited from a TMS test pulse of the motor cortex [25, 36]. Each single pulse of TMS over the cerebellum produces a short decrease in cortical excitability 5 to $6 \mathrm{~ms}$ later, an effect and a latency that are physiologically consistent with TMS stimulating the Purkinje cells of the cerebellum. rTMS of the cerebellum is also effective in modulating motor cortex excitability [37] and disrupting simple and coordinated movements of the hand $[38,39]$. The effect on motor cortex excitability of rTMS of the cerebellum is frequency dependent. Low-frequency stimulation induces an increase in excitability of the cerebello-thalamocortical pathway measured with probe trials at the motor cortex [40] which indicated that low-frequency rTMS as used in the current study causes a reduction in Purkinje cell excitability (see [41]).

Is the site of interference somewhere else other than the $O M V$ ? It is possible that the site at which the rTMS interferes with saccadic adaptation is at a locus other than the OMV. Firstly, we must consider whether the site of stimulation is outside the cerebellum. TMS stimulation over the back of the head can stimulate the visual cortex. Classically, stimulation of the visual cortex can produce visual phosphenes or temporarily mask visual perception. In these studies, the site of stimulation is dorsal to the inion (by 2-4 cm). It is unlikely that we stimulated the visual cortex as none of our subjects reported any visual phenomena, and the site of stimulation was below the visual cortex (see Fig. 1). It is also possible that stimulation of the musculature of the neck could have some non-specific effects that could confound the findings in the study. We think this unlikely because in terms of induced muscle contraction and sensory stimulation the two TMS conditions $(45 \%$ and $55 \%)$ we used were very similar but produced a significantly different effect of saccadic adaptation. This would not be expected if the effect was due to scalp or neck muscle activation, both on which have relatively low threshold compared to the cerebellum. The cerebellar hemispheres are greatly expanded in humans, with the paravermal cerebellum extending beyond the vermis, while the OMV is deeper and beneath the surrounding paravermis. Though gross anatomy [42] and modern imaging [43] confirm that the paravermal hemispheres partially occlude the cerebellar vermis, there is still a clear line of sight to the OMV in the intact brain. The areas of paravermal cerebellum around the OMV most likely to be coincidentally stimulated by rTMS in our study are crus I and II (see Fig. 1). Microelectrode electrical stimulation of crus I and II of a monkey elicits ipsiversive eye movements [1], so it seems feasible that manipulation of this area may lead to a deficit in saccadic adaptation. However, lesions of the OMV that spare crus I and II in the monkey cause a non-recoverable deficit in saccadic adaptation, suggesting that crus I and II alone without the OMV cannot produce saccadic adaption. We also chose to use a double-cone stimulating coil. The configuration of these coils is such that they induce electric fields at greater depth than traditional flat figure-of-eight coils [44]. They have been successfully used to stimulate the leg area of the motor cortex that lie approximately $3-4 \mathrm{~cm}$ below the scalp [45], a similar depth as the OMV. Together, these facts lead us to suggest that, although TMS may stimulate sites in the cerebellum other than the OMV, it is specifically rTMS of the OMV that causes the deficit in saccadic adaptation seen in our experiment.

Low-frequency rTMS would have interrupted the normal function of the OMV by suppressing the excitability of the cells therein and therefore decrease the output of the cerebellar cortex to the deep cerebellar nuclei. Our data are comparable to studies in non-human primates where ablation of the OMV produces a permanent inability to adapt saccade size [10] and patient studies that have demonstrated that individuals with focal cerebellar lesions or cerebellar degeneration are less able to adapt saccades [27-29]. Our data also substantiate human imaging data that demonstrate a correlation between increased blood flow in the OMV with saccadic adaptation [46, 47] and corroborate single-cell studies which show that the population cell firing in OMV changes in a way that could drive adaptation [30]. Barash et al. [10] demonstrated that ablation of the OMV of the macaque cerebellum produced a profound inability to adjust the size of saccades during an adaptation task. The deficit was permanent for the duration of the post-lesion period (up to 1 year). Our experiment 
demonstrates that rTMS of the posterior cerebellum produces a qualitatively similar deficit, though the deficit following rTMS is neither total nor permanent. An increase in intensity of rTMS from $45 \%$ to $55 \%$ decreased the ability to adapt saccades, suggesting that an increase in stimulation increases the amount of suppression of the area of cerebellum under the coil. A similar relationship between increasing rTMS intensities and an increase in suppression of activity has been demonstrated in the motor cortex [48]. Our results suggest that rTMS to the posterior cerebellum produces a suppression of the normal function of the OMV that is qualitatively similar to a lesion and that this dysfunction increases with the intensity of rTMS.

In summary, evidence to date of the role of the OMV in saccadic adaptation in humans has been from either patient data [27-29, 49] or brain imaging experiments [46, 47]. In this study, we provide further evidence of the role of the cerebellum in saccadic adaptation. We show that manipulation of the activity of the posterior cerebellum including the OMV in healthy human subjects affects motor learning in voluntary eye movements and demonstrates the feasibility of using TMS to probe the role of the cerebellum in oculomotor control.

Acknowledgments This work was supported by the Wellcome Trust.

Conflict of interest The authors declare that they have no conflict of interest.

Open Access This article is distributed under the terms of the Creative Commons Attribution Noncommercial License which permits any noncommercial use, distribution, and reproduction in any medium, provided the original author(s) and source are credited.

\section{References}

1. Ron S, Robinson D. Eye movements evoked by cerebellar stimulation in the alert monkey. J Neurophysiol. 1973;36(6):1004-22.

2. Noda H, Fujikado T. Involvement of Purkinje cells in evoking saccadic eye movements by microstimulation of the posterior cerebellar vermis of monkeys. J Neurophysiol. 1987;57(5):1247-61.

3. Fujikado T, Noda H. Saccadic eye movements evoked by microstimulation of lobule VII of the cerebellar vermis of macaque monkeys. J Physiol. 1987;394:573-94.

4. Ohtsuka K, Noda H. Saccadic burst neurons in the oculomotor region of the fastigial nucleus of macaque monkeys. J Neurophysiol. 1991;65(6):1422-34.

5. Fuchs A, Robinson F, Straube A. Role of the caudal fastigial nucleus in saccade generation. I. Neuronal discharge pattern. J Neurophysiol. 1993;70(5):1723-40.

6. Ritchie L. Effects of cerebellar lesions on saccadic eye movements. J Neurophysiol. 1976;39(6):1246-56.

7. Optican L, Robinson D. Cerebellar-dependent adaptive control of primate saccadic system. J Neurophysiol. 1980;44(6):1058-76.
8. Goldberg M, Musil S, Fitzgibbon E, Smith M, Olson C. The role of the cerebellum in the control of saccadic eye-movements. Role Cerebellum Basal Ganglia Voluntary Movement. 1993;1024:203-11.

9. Robinson F, Fuchs A, Noto C. Cerebellar influences on saccade plasticity. Ann NY Acad Sci. 2002;956:155-63.

10. Barash S, Melikyan A, Sivakov A, Zhang M, Glickstein M, Thier P. Saccadic dysmetria and adaptation after lesions of the cerebellar cortex. J Neurosci. 1999;19(24):10931-9.

11. Takagi M, Zee D, Tamargo R. Effects of lesions of the oculomotor vermis on eye movements in primate: saccades. J Neurophysiol. 1998;80(4):1911-31.

12. Carpenter R. Cerebellectomy and the transfer function of the vestibulo-ocular reflex in the decerebrate cat. Proc R Soc Lond B Biol Sci. 1972;181(1065):353-74.

13. Robinson D. Adaptive gain control of vestibulo-ocular reflex by the cerebellum. J Neurophysiol. 1976;39(5):954-69.

14. McCormick D, Thompson R. Cerebellum: essential involvement in the classically conditioned eyelid response. Science. 1984;223 (4633):296-9.

15. Yeo $\mathrm{CH}$, Hardiman MJ, Glickstein M. Discrete lesions of the cerebellar cortex abolish the classically conditioned nictitating membrane response of the rabbit. Behav Brain Res. 1984;13(3):261-6.

16. Baizer J, Kralj-Hans I, Glickstein M. Cerebellar lesions and prism adaptation in macaque monkeys. J Neurophysiol. 1999;81 (4):1960-5.

17. Baizer J, Glickstein M. Proceedings: role of cerebellum in prism adaptation. J Physiol (Lond). 1974;236(1):34P-5.

18. Marr D. A theory of cerebellar cortex. J Physiol (Lond). 1969;202 (2):437-70.

19. Albus J. A theory of cerebellar function. Math Biosci. 1971;10(12):61-25.

20. Soetedjo R, Fuchs A. Complex spike activity of Purkinje cells in the oculomotor vermis during behavioral adaptation of monkey saccades. J Neurosci. 2006;26(29):7741-55.

21. Catz N, Dicke P, Thier P. Cerebellar complex spike firing is suitable to induce as well as to stabilize motor learning. Curr Biol. 2005;15(24):2179-89.

22. Pélisson D, Alahyane N, Panouillères M, Tilikete C. Sensorimotor adaptation of saccadic eye movements. Neurosci Biobehav Rev. 2010;34(8):1103-20.

23. McLaughlin S. Parametric adjustment in saccadic eye movements. Percept Psychophys. 1967;2:359-62.

24. Siebner H, Rothwell J. Transcranial magnetic stimulation: new insights into representational cortical plasticity. Exp Brain Res. $2003 ; 148(1): 1-16$.

25. Ugawa Y, Uesaka Y, Terao Y, Hanajima R, Kanazawa I. Magnetic stimulation over the cerebellum in humans. Ann Neurol. 1995;37 (6):703-13.

26. Chen RM, Gerloff C, Celnik P, Wassermann EM, Hallett M, et al. Depression of motor cortex excitability by low-frequency transcranial magnetic stimulation. Neurology. 1997;48(5):1398-403.

27. Golla H, Tziridis K, Haarmeier T, Catz N, Barash S, Thier P. Reduced saccadic resilience and impaired saccadic adaptation due to cerebellar disease. Eur J Neurosci. 2008;27(1):132-44.

28. Straube A, Deubel H, Ditterich J, Eggert T. Cerebellar lesions impair rapid saccade amplitude adaptation. Neurology. 2001;57(11):2105-8.

29. Choi K, Kim H, Cho B, Kim J. Saccadic adaptation in lateral medullary and cerebellar infarction. Exp Brain Res. 2008;188 (3):475-82.

30. Catz N, Dicke P, Thier P. Cerebellar-dependent motor learning is based on pruning a Purkinje cell population response. Proc Natl Acad Sci U S A. 2008;105(20):7309-14.

31. Soetedjo R, Kojima Y, Fuchs AF. Complex spike activity in the oculomotor vermis of the cerebellum: a vectorial error signal for saccade motor learning? J Neurophysiol. 2008;100 (4):1949-66. 
32. Hashimoto M, Ohtsuka K. Transcranial magnetic stimulation over the posterior cerebellum during visually guided saccades in man. Brain. 1995;118(5):1185-93.

33. Ohtsuka K, Enoki T. Transcranial magnetic stimulation over the posterior cerebellum during smooth pursuit eye movements in man. Brain. 1998;121(3):429-35.

34. Zangemeister WH, Nagel M. Transcranial magnetic stimulation over the cerebellum delays predictive head movements in the coordination of gaze. Acta Otolaryngol Suppl. 2001;545: $140-4$.

35. Miall R, Christensen L, Cain O, Stanley J. Disruption of state estimation in the human lateral cerebellum. PLoS Biol. 2007;5(11): e316.

36. Werhahn KJ, Taylor J, Ridding M, Meyer BU, Rothwell JC. Effect of transcranial magnetic stimulation over the cerebellum on the excitability of human motor cortex. Electroencephalogr Clin Neurophysiol. 1996;101(1):58-66.

37. Oliveri $\mathrm{M}$, Koch $\mathrm{G}$, Torriero $\mathrm{S}$, Caltagirone $\mathrm{C}$. Increased facilitation of the primary motor cortex following $1 \mathrm{~Hz}$ repetitive transcranial magnetic stimulation of the contralateral cerebellum in normal humans. Neurosci Lett. 2005;376(3):188-93.

38. Miall R, Christensen L. The effect of rTMS over the cerebellum in normal human volunteers on peg-board movement performance. Neurosci Lett. 2004;371(2-3):185-9.

39. Del Olmo M, Cheeran B, Koch G, Rothwell J. Role of the cerebellum in externally paced rhythmic finger movements. J Neurophysiol. 2007;98(1):145-52.

40. Langguth B, Eichhammer P, Zowe M, Landgrebe M, Binder H, Sand $\mathrm{P}$, et al. Modulating cerebello-thalamocortical pathways by neuro- navigated cerebellar repetitive transcranial stimulation (rTMS). Clin Neurophysiol. 2008;38(5):289-95.

41. Daskalakis ZJ, Paradiso GO, Christensen BK, Fitzgerald PB, Gunraj $\mathrm{C}$, Chen R. Exploring the connectivity between the cerebellum and motor cortex in humans. J Physiol. 2004;557(2):689-700.

42. Larsell $\mathrm{O}$. The comparative anatomy and histology of the cerebellum. Minneapolis: University of Minnesota Press; 1967.

43. Diedrichsen J. A spatially unbiased atlas template of the human cerebellum. Neuroimage. 2006;33(1):127-38.

44. Roth Y, Zangen A, Hallett M. A coil design for transcranial magnetic stimulation of deep brain regions. J Clin Neurophysiol. 2002;19(4):361-70.

45. Terao Y, Ugawa Y, Hanajima R, Machii K, Furubayashi T, Mochizuki $\mathrm{H}$, et al. Predominant activation of I1-waves from the leg motor area by transcranial magnetic stimulation. Brain Res. 2000;859(1):137-46.

46. Desmurget M, Pelisson D, Urquizar C, Prablanc C, Alexander G, Grafton S. Functional anatomy of saccadic adaptation in humans. Nat Neurosci. 1998;1(6):524-8.

47. Desmurget M, Pelisson D, Grethe J, Alexander G, Urquizar C, Prablanc $\mathrm{C}$, et al. Functional adaptation of reactive saccades in humans: a PET study. Exp Brain Res. 2000;132(2):243-59.

48. Lang N, Harms J, Weyh T, Lemon R, Paulus W, Rothwell J, et al. Stimulus intensity and coil characteristics influence the efficacy of rTMS to suppress cortical excitability. Clin Neurophysiol. 2006;117(10):2292-301.

49. Gaymard B, Rivaud-Péchoux S, Yelnik J, Pidoux B, Ploner C. Involvement of the cerebellar thalamus in human saccade adaptation. Eur J Neurosci. 2001;14(3):554-60. 